\title{
Geometric Formation Control for Autonomous Underwater Vehicles
}

\author{
Huizhen Yang and Fumin Zhang
}

\begin{abstract}
This paper presents a novel approach based on Jacobi shape theory and geometric reduction for formation control of autonomous underwater vehicles (AUVs). We consider a three degree-of-freedom (DOF) dynamic model for the horizontal motion of each AUV that has control inputs over surge force and yaw moment. By using the Jacobi transform, the horizontal dynamics of AUVs are expressed as dynamics for formation shape, formation motion and vehicle orientation. The system decouples when additional symmetries in vehicle design are presented. Hence formation shape controllers, formation motion controllers, and vehicle orientation controllers can be designed separately. This approach reduces the complexity of formation controllers. We use the model for ODIN as an example to demonstrate the controller design process. Simulation results show the effectiveness of the controllers.
\end{abstract}

\section{INTRODUCTION}

The formation control problems of multiple AUVs have received much recent attention with applications in oceanographic research, seafloor survey, underwater archeology and meteorology. Cooperative control enables the vehicles to combine sensor data and create smarter behaviors than those of a single vehicle.

A common practice in some of the existing results for formation control is to simplify the motion dynamics of an individual vehicle or robot to a second-order particle model [1]-[7]. Formation control becomes more challenging if more practical and complex dynamics are concerned. Various methods have been developed to answer this challenge. A leader-follower formation control scheme for autonomous helicopters is investigated in [8] by applying the slidingmode controller design method, where a 6 DOF dynamic model is considered. In [9], a dynamic model of the AUV ODIN [10] is used to design a proportional-derivative controller for formation control. A 3 DOF horizontal model for AUVs is used in [11] and [12]. In [11], the model has decoupled sway and yaw motion. A virtual vehicle is employed to provide a reference trajectory and velocity for the followers with their tracking controllers designed using the back-stepping method. In [12], the horizontal dynamic model of a torpedo type AUV is described using a general nonlinear mapping, and formation controllers are designed based on artificial potential functions. A cross-track control scheme based on Line of Sight (LOS) guidance law is presented to make the AUVs follow a given straight line

H. Yang is with the School of Marine, Northwestern Polytechnical University, China huizhenyang1962@hotmail. com. Her research is supported by the "Ao Xiang Star Plan".

F. Zhang is with the School of Electrical and Computer Engineering, Georgia Institute of Technology,USA. fumindece.gatech.edu. His research is supported by ONR grants N00014-08-1-1007 and N0001409-1-1074, and NSF grants ECCS-0841195 and CNS-0931576. and constitute a desired formation in [13], where a 5 DOF dynamic model with independent control inputs in surge, pitch and yaw is considered. Similar approach is extended to surface vessels described by a 3 DOF dynamic model where the surge dynamics are decoupled from the steering dynamics [14]. A cooperative controller based on discrete time Kuramoto models is designed to integrate communication and control for multiple vehicles [15]. Experimental results on the University of Washington Fin-actuated Autonomous Underwater System(UMMFAUS) are reported in [16].

One of the major difficulties of formation control for AUVs is that the collected dynamics of all vehicles are more complex than the non-trivial single vehicle dynamics. The reviewed existing methods design formation controllers for the collected dynamics directly. The vehicle dynamics lead to results that are difficult to be justified theoretically. In this paper, we employ an approach based on geometric reductions for formation control of multiple AUVs. The approach expresses the formation dynamics as a deformable body by using the Jacobi coordinates that has been previously applied to formation control for particles in Zhang's works [4]-[6]. In this paper, we extend the methods to control of AUV formations. The key benefit of this approach is that the dynamics of the formation shape and formation center are explicitly revealed. Furthermore, in some cases with additional symmetry, the collected motion dynamics are decomposed into dynamics for the formation shape, the formation center, and the vehicle orientation. Each set of decoupled dynamics is simpler than the collected dynamics, hence controller design for the decoupled dynamics is simplified.

We apply our design methods to the nonlinear horizontal dynamic model of ODIN [10], an autonomous underwater vehicle designed by the University of Hawaii. We show that each decoupled system is linear, hence controllers can be designed using linear state feedback.

The organization of this paper is as follows. In Section II, the horizontal dynamic equations of a single AUV are reviewed. We derive the formation dynamic of multiple AUVs through Jacobi transform in Section III. Formation shape controllers, formation motion controllers and AUV orientation controllers for ODINs are designed in Section IV. Numerical simulation results are given in Section V. Summary and discussions are presented in Section VI.

\section{DYNAMIC MODEL OF A SINGLE AUV}

We consider a 3 DOF horizontal motion model that describes surge, sway and yaw motion for an AUV [17]. We define $\eta=[x, y, \psi]^{T}$ where $[x, y]^{T}$ represents the vehicle position in the horizontal plane and $\psi$ is the yaw angle, and 
use $v=[u, v, r]^{T}$ for the body-fixed linear velocity vector for surge, sway and yaw. We assume that the AUV is neutrally buoyant with three planes of symmetry. For simplicity, we only consider the linear hydrodynamic damping forces and moments. We select the origin of the body frame of the vehicle to coincide with the center of gravity. The horizontal dynamics can be expressed as follows:

$$
\dot{\eta}=R_{b}^{I}(\psi) v
$$

and

$$
M \dot{v}+C(v) v+D v=\tau^{\prime}
$$

where

$$
\begin{aligned}
& R_{b}^{I}(\psi)=\left[\begin{array}{ccc}
\cos \psi & -\sin \psi & 0 \\
\sin \psi & \cos \psi & 0 \\
0 & 0 & 1
\end{array}\right], \\
& M=\left[\begin{array}{ccc}
m-X_{\dot{u}} & 0 & 0 \\
0 & m-Y_{\dot{v}} & 0 \\
0 & 0 & I_{z}
\end{array}\right], \\
& C(v)=\left[\begin{array}{ccc}
0 & 0 & -m v+Y_{\dot{v}} v \\
0 & 0 & m u-X_{\dot{u}} u \\
m v-Y_{\dot{v}} v & -m u+X_{\dot{u}} u & 0
\end{array}\right], \\
& D=\left[\begin{array}{ccc}
-X_{u} & 0 & 0 \\
0 & -Y_{v} & 0 \\
0 & 0 & -N_{r}
\end{array}\right], \tau^{\prime}=\left[\begin{array}{c}
\tau_{x} \\
\tau_{y} \\
\tau_{\psi}
\end{array}\right] .
\end{aligned}
$$

The matrix $R_{b}^{I}(\psi)$ is the rotation matrix from the body frame to the inertial frame. $M$ denotes the inertia matrix. $C(v)$ contains Coriolis and centrifugal force terms, and $D$ is the hydrodynamic damping matrix. $\tau^{\prime}$ is the vector of control inputs where $\tau_{x}$ is the surge force, $\tau_{y}$ is the sway force, and $\tau_{\psi}$ is the yaw moment. $X_{u}, Y_{v}, N_{r}, X_{\dot{u}}, Y_{\dot{v}}$ and $I_{z}$ are model parameters.

Let $p=[x, y]^{T}$ and $\gamma=[u, v]^{T}$. We can rewrite the position and orientation transformation described in equation (1) as follows:

$$
\begin{gathered}
\dot{p}=R(\psi) \gamma \\
\dot{\psi}=r
\end{gathered}
$$

where

$$
R(\psi)=\left[\begin{array}{cc}
\cos \psi & -\sin \psi \\
\sin \psi & \cos \psi
\end{array}\right]
$$

has the properties that $R^{T}(\psi) R(\psi)=I$ for all $\psi$, and

$$
\dot{R}(\psi)=R(\psi) S(\dot{\psi})
$$

where

$$
S(\dot{\psi})=\left[\begin{array}{cc}
0 & -\dot{\psi} \\
\dot{\psi} & 0
\end{array}\right]
$$

is skew-symmetric.

Next, decomposing the equation (2), we have

$$
M_{1} \dot{\gamma}+N(r) \gamma=\tau
$$

and

$$
\dot{r}=-\frac{\left(X_{\dot{u}}-Y_{\dot{v}}\right)}{I_{z}} u v+\frac{N_{r}}{I_{z}} r+\frac{1}{I_{z}} \tau_{\psi}
$$

where,

$$
\begin{gathered}
M_{1}=\left[\begin{array}{cc}
m-X_{\dot{u}} & 0 \\
0 & m-Y_{\dot{v}}
\end{array}\right] \\
N(r)=\left[\begin{array}{cc}
-X_{u} & \left(-m+Y_{\dot{v}}\right) r \\
\left(m-X_{\dot{u}}\right) r & -Y_{v}
\end{array}\right] \\
\tau=\left[\begin{array}{c}
\tau_{x} \\
\tau_{y}
\end{array}\right] .
\end{gathered}
$$

Equation (9) implies that

$$
\dot{\gamma}=M_{1}^{-1}(\tau-N(r) \gamma) .
$$

Taking derivatives on both sides of equation (4) yields

$$
\ddot{p}=\dot{R}(\psi) \gamma+R(\psi) \dot{\gamma}=R(\psi) S(r) \gamma+R(\psi) \dot{\gamma} .
$$

Substituting equation (7) and (12) into (13), we have

$$
\ddot{p}=R(\psi)\left[S(r)-M_{1}^{-1} N(r)\right] R^{-1}(\psi) \dot{p}+R(\psi) M_{1}^{-1} \tau .
$$

Define

$$
G(\psi, r)=R(\psi)\left[S(r)-M_{1}^{-1} N(r)\right] R^{-1}(\psi)
$$

and

$$
H(\psi)=R(\psi) M_{1}^{-1} .
$$

Then equation (14) can be rewritten as:

$$
\ddot{p}=G(\psi, r) \dot{p}+H(\psi) \tau .
$$

Equation (17) and the equations of vehicle orientation described by equation (5) and (10) are nonlinear equations about state variables $(\psi, r, p, \dot{p})$ with control inputs $\tau$ and $\tau_{\psi}$. It can be observed that equation (17) differs from a second order particle model in that the orientation and angular speed affect the translation dynamics. If multiple vehicles are considered, then the differences in the orientation and angular speed among the vehicles make the formation control problem more challenging than the case when all particles are considered identical.

\section{FORMATION DYNAMICS}

The entire formation of $N$ AUVs can be viewed as a deformable body. Jacobi vectors can be defined to describe the shape and orientation of a deformable body [6]. Suppose the positions of the AUVs are described by $p_{i}=\left[x_{i}, y_{i}\right]^{T}, i=$ $1,2, \ldots, N$. Then the Jacobi vectors are defined by a linear transform $\Phi$ that produces the following equation:

$$
\left[\rho_{1}, \rho_{2}, \ldots, \rho_{N-1}, q_{c}\right]^{T}=\Phi\left[p_{1}, p_{2}, \ldots, p_{N}\right]^{T}
$$

where $\rho_{i}(i=1,2, \ldots, N-1)$ are the Jacobi vectors and $q_{c}$ is the formation center, defined by

$$
q_{c}=\frac{1}{N} \sum_{i=1}^{N} p_{i} .
$$

The following realization of the transform $\Phi$ may be used when $N=3$ :

$$
\begin{aligned}
& \rho_{1}=\frac{1}{\sqrt{2}}\left(p_{2}-p_{1}\right) \\
& \rho_{2}=p_{3}-\frac{1}{2}\left(p_{1}+p_{2}\right),
\end{aligned}
$$


as illustrated by Figure 1 . If we can control $\rho_{1} \rightarrow \rho_{1 d}$ and $\rho_{2} \rightarrow \rho_{2 d}$ as $t \rightarrow \infty$, where $\rho_{1 d}$ and $\rho_{2 d}$ are desired constant vectors, then the three vehicles will converge to a constant triangular formation.

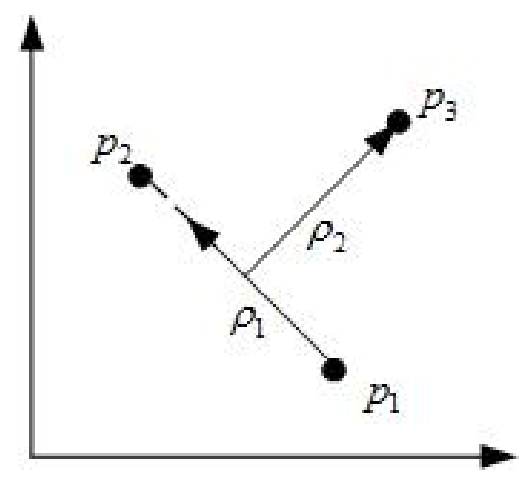

Fig. 1. Definition of Jacobi vectors for three vehicles.

Taking derivatives on both sides of equation (18), we have

$$
\left[\dot{p}_{1}, \dot{p}_{2}, \ldots, \dot{p}_{N}\right]^{T}=\Phi^{-1}\left[\dot{\rho}_{1}, \dot{\rho}_{2}, \ldots, \dot{\rho}_{N-1}, \dot{q}_{c}\right]^{T}
$$

Then we derive dynamic equations of the Jacobi vectors and the formation center vector by taking the second order derivative of equation (18) and substituting equations (14) and (21) into the following equation.

$$
\begin{aligned}
& {\left[\begin{array}{c}
\ddot{\rho}_{1} \\
\vdots \\
\ddot{\rho}_{N-1} \\
\ddot{q}_{c}
\end{array}\right]=\Phi\left[\begin{array}{c}
\ddot{p}_{1} \\
\vdots \\
\ddot{p}_{N}
\end{array}\right]=\Phi\left[\begin{array}{c}
G\left(\psi_{1}, r_{1}\right) \dot{p}_{1}+H\left(\psi_{1}\right) \tau_{1} \\
\vdots \\
G\left(\psi_{N}, r_{N}\right) \dot{p}_{N}+H\left(\psi_{N}\right) \tau_{N}
\end{array}\right]} \\
& =\Phi\left[\begin{array}{cc}
G\left(\psi_{1}, r_{1}\right) & \\
+\Phi &
\end{array}\right] \Phi^{-1}\left[\begin{array}{c}
\dot{\rho}_{1} \\
\vdots \\
\dot{\rho}_{N-1} \\
\dot{q}_{c}
\end{array}\right] \\
& \left.+\begin{array}{c}
H\left(\psi_{1}\right) \tau_{1} \\
\vdots \\
H\left(\psi_{N}\right) \tau_{N}
\end{array}\right] .
\end{aligned}
$$

Define a state vector $\mathbf{X}=\left[\rho_{1}, \ldots, \rho_{N-1}, q_{c}\right]^{T}$ and let

$$
\mathbf{G}=\left[\begin{array}{lll}
G\left(\psi_{1}, r_{1}\right) & & \\
& \ldots & \\
& & G\left(\psi_{N}, r_{N}\right)
\end{array}\right]
$$

Furthermore, let

$$
\mathbf{A}=\Phi \mathbf{G} \Phi^{-1}
$$

Define

$$
u_{i}=H\left(\psi_{i}\right) \tau_{i}
$$

and let

$$
\mathbf{U}=\left[u_{1}, \ldots, u_{N}\right]^{T} .
$$

The dynamic equations of the formation is now

$$
\ddot{\mathbf{X}}=\mathbf{A} \dot{\mathbf{X}}+\Phi \mathbf{U}
$$

Equation (27), equation (5) and equation (10) describe the formation dynamics as well as the steering dynamics. The block diagonal matrix $\mathbf{G}$ plays an important role in the formation dynamics in that it determines whether the formation shape dynamics described by the Jacobi shape vectors $\rho_{i}$ and the formation center dynamics described by the center vector $q_{c}$ are decoupled. For each block of $\mathbf{G}$, we have

$$
\begin{aligned}
G\left(\psi_{i}, r_{i}\right) & =R\left(\psi_{i}\right)\left[S\left(r_{i}\right)-M_{1}^{-1} N\left(r_{i}\right)\right] R^{-1}\left(\psi_{i}\right) \\
& =\left[\begin{array}{ll}
g_{11} & g_{12} \\
g_{21} & g_{22}
\end{array}\right]
\end{aligned}
$$

where,

$$
\begin{aligned}
g_{11}= & \frac{X_{u}}{m-X_{\dot{u}}} \cos ^{2} \psi_{i}+\left(\frac{m-X_{\dot{u}}}{m-Y_{\dot{v}}}-\frac{m-Y_{\dot{v}}}{m-X_{\dot{u}}}\right) r_{i} \sin \psi_{i} \cos \psi_{i}+ \\
& \frac{Y_{v}}{m-Y_{\dot{v}}} \sin ^{2} \psi_{i} \\
g_{12}= & \left(\frac{X_{u}}{m-X_{\dot{u}}}-\frac{Y_{v}}{m-Y_{\dot{v}}}\right) \sin \psi_{i} \cos \psi_{i}+\frac{m-X_{\dot{u}}}{m-Y_{\dot{v}}} r_{i} \sin ^{2} \psi_{i}+ \\
& \frac{m-Y_{\dot{v}}}{m-X_{\dot{u}}} r_{i} \cos ^{2} \psi_{i}-r_{i} \\
g_{21}= & \left(\frac{X_{u}}{m-X_{\dot{u}}}-\frac{Y_{v}}{m-Y_{\dot{v}}}\right) \sin \psi_{i} \cos \psi_{i}-\frac{m-X_{\dot{u}}}{m-Y_{\dot{v}}} r_{i} \cos ^{2} \psi_{i}- \\
& \frac{m-Y_{\dot{v}}}{m-X_{\dot{u}}} r_{i} \sin ^{2} \psi_{i}+r_{i} \\
g_{22}= & \frac{X_{u}}{m-X_{\dot{u}}} \sin ^{2} \psi_{i}+\left(\frac{m-Y_{\dot{v}}}{m-X_{\dot{u}}}-\frac{m-X_{\dot{u}}}{m-Y_{\dot{v}}}\right) r_{i} \sin \psi_{i} \cos \psi_{i}+ \\
& \frac{Y_{v}}{m-Y_{\dot{v}}} \cos ^{2} \psi_{i} .
\end{aligned}
$$

We see that if we ignore the drag forces i.e. letting $X_{u}$ and $Y_{v}$ be trivial, then $G\left(\psi_{i}, r_{i}\right)$ is trivial for all $i=1,2, \ldots, N$. Hence the formation shape and formation center dynamics are decoupled. But if the drag forces can not be ignored, the dynamics are not decoupled in general.

In the next section we will show that for the ODIN vehicle [10], due to additional symmetry in the vehicle design, the dynamics of the formation shape and formation center are decoupled when drag forces are considered. We can then design the formation shape and the formation center controllers separately using linear state feedback.

\section{Formation CONTROL FOR ODINs}

In this section, we design formation controllers for ODINs. We show that the formation shape and center dynamics are decoupled due to properties of the hydrodynamic parameters of ODIN. Then we design the formation shape and formation motion control using linear state feedback. Assuming that the ODIN is controlled by the surge force and steering control but no sway forces are applied, we follow the inner-outer loop design approach to separate the design of the surge control and the steering control. 


\section{A. Formation dynamics of ODINs}

The hydrodynamic parameters of ODIN satisfy $Y_{\dot{v}}=X_{\dot{u}}$ and $Y_{v}=X_{u}$. Therefore we get $M^{-1}=\left[\begin{array}{cc}a & 0 \\ 0 & a\end{array}\right]$, where $a=$ $\frac{1}{m-X_{\dot{u}}}=\frac{1}{m-Y_{\dot{v}}}$. Furthermore,

$$
G\left(\psi_{i}, r_{i}\right)=\left[\begin{array}{cc}
a X_{u} & 0 \\
0 & a X_{u}
\end{array}\right]=a X_{u} I_{2}
$$

which is a constant $2 \times 2$ diagonal matrix. Hence $\mathbf{G}=a X_{u} I_{2 N}$. The system matrix of formation dynamic equation (27) becomes a constant diagonal matrix. i.e.

$$
A=\Phi \mathbf{G} \Phi^{-1}=a X_{u} I_{2 N} .
$$

Now define

$$
F=\left[f_{1}, f_{2}, \cdots, f_{c}\right]^{T}=\Phi \mathbf{U} .
$$

Then the formation shape and center motion systems are simplified to:

$$
\begin{aligned}
& \ddot{\rho}_{i}=a X_{u} I_{2} \dot{\rho}_{i}+f_{i}, i=1,2, \cdots, N-1 \\
& \ddot{q}_{c}=a X_{u} I_{2} \dot{q}_{c}+f_{c} .
\end{aligned}
$$

And the vehicle orientation dynamic becomes linear as described by the following equation:

$$
\begin{aligned}
& \dot{\psi}=r \\
& \dot{r}=\frac{N_{r}}{I_{z}} r+\frac{1}{I_{z}} \tau_{\psi} .
\end{aligned}
$$

\section{B. Formation Shape and Center Controller}

For the linear formation shape subsystems

$$
\ddot{\rho}_{i}=a X_{u} I_{2} \dot{\rho}_{i}+f_{i}, i=1,2, \cdots, N-1 .
$$

We can design control force by using linear state feedback.

Define an augmented state vector $\bar{X}_{i}=\left[\rho_{i}, \dot{\rho}_{i}\right]^{T}$. The augmented state equation of $\bar{X}_{i}$ can be described as the following:

$$
\dot{\bar{X}}_{i}=\bar{A} \bar{X}_{i}+\bar{B} f_{i}
$$

where

$$
\bar{A}=\left[\begin{array}{cc}
0 & I_{2} \\
0 & a X_{u} I_{2}
\end{array}\right], \bar{B}=\left[\begin{array}{c}
0 \\
I_{2}
\end{array}\right] .
$$

Define the tracking error vector $E_{i}=\bar{X}_{i}-\bar{X}_{i d}$, where $\bar{X}_{i d}$ is the desired shape. So the state equation of this error vector is as following:

$$
\dot{E}_{i}=\bar{A} E_{i}+\bar{A} \bar{X}_{i d}+\bar{B} f_{i}-\dot{\bar{X}}_{i d} .
$$

Let

$$
v_{i}=a X_{u} \dot{\rho}_{i d}+f_{i}-\ddot{\rho}_{i d}
$$

Then the state equation of $E_{i}$ can be written as:

$$
\dot{E}_{i}=\bar{A} E_{i}+\bar{B} v_{i}
$$

Because the rank of controllability matrix

$$
S_{c}=\left[\bar{B} \mid \bar{A} \bar{B}, \bar{A}^{2} \bar{B}\right]=\left[\begin{array}{cccccc}
0 & 0 & 1 & 0 & a X_{u} & 0 \\
0 & 0 & 0 & 1 & 0 & a X_{u} \\
1 & 0 & a X_{u} & 0 & \left(a X_{u}\right)^{2} & 0 \\
0 & 1 & 0 & a X_{u} & 0 & \left(a X_{u}\right)^{2}
\end{array}\right]
$$

is 4 , the controllability of system $[\bar{A}, \bar{B}]$ is verified. Then we can design the state feedback gain $K^{\rho}$ to place all the eigenvalues of $\left(\bar{A}-\bar{B} K^{\rho}\right)$ on the LHP. Then the control law

$$
v_{i}=-K^{\rho} E_{i}
$$

can guarantee the goal $E_{i} \rightarrow 0$ as $t \rightarrow \infty$, i.e. $\bar{X}_{i} \rightarrow \bar{X}_{i d}$ with an exponential rate of convergence. This implies $\rho_{i} \rightarrow \rho_{i d}$ and $\dot{\rho}_{i} \rightarrow \dot{\rho}_{i d}$, where $\rho_{i d}$ are desired configureation in Jacobi coordinates. Therefore, if the desired shape is constant, then we get the formation shape control forces:

$$
f_{i}=-k_{1}^{\rho}\left(\rho_{i}-\rho_{i d}\right)-k_{2}^{\rho} \dot{\rho}_{i} i=1,2, \ldots, N-1
$$

where $k_{1}^{\rho}, k_{2}^{\rho}>0$ are controller gains.

The control for the formation center motion can be designed in the same way as the shape controllers. To track a desired trajectory given by $q_{c d}(t)$, the control force for the center can be

$$
f_{c}=\ddot{q}_{c d}-a X_{u} \dot{q}_{c d}-k_{1}^{q}\left(q_{c}-q_{c d}\right)-k_{2}^{q}\left(\dot{q}_{c}-\dot{q}_{c d}\right)
$$

where $k_{1}^{q}, k_{2}^{q}>0$ are controller gains.

\section{Orientation Controller}

We suppose that the vehicle is controlled by surge force and steering torque by no sway force i.e. $\tau_{y}=0$. Then we define

$$
H\left(\psi_{i}\right) \tau_{i}=\left[\begin{array}{l}
u_{i 1} \\
u_{i 2}
\end{array}\right]
$$

where

$$
u_{i 1}=a \tau_{i x} \cos \psi_{i}, u_{i 2}=a \tau_{i x} \sin \psi_{i}
$$

A inner-outer loop controller design method can then be followed by assuming that the steering dynamics can be controlled faster than the translation dynamics. Note that if the sway force control was available, then such innerouter loop design procedure would not be necessary and the steering controller design problem would be completely decoupled from the formation controller design.

Once we know the control force $f_{i}, i=1,2, \cdots, N-1$ and $f_{c}$, we can compute the desired value of $\mathbf{U}$ by $\mathbf{U}=\Phi^{-1} F$. According to equation (46), the surge forces for each ODIN are

$$
\tau_{i x}=\frac{u_{i 1}}{a \cos \psi_{i d}}=\frac{u_{i 2}}{a \sin \psi_{i d}}
$$

where the desired yaw angle $\psi_{\text {id }}$ should be

$$
\psi_{i d}=\operatorname{atan} 2\left(u_{i 1}, u_{i 2}\right)
$$

Therefore, from the computed control forces $u_{i 1}$ and $u_{i 2}$, the surge force and the yaw angle are uniquely determined. We design the vehicle orientation controller to achieve $\psi_{i} \rightarrow \psi_{i d}$ as $t \rightarrow \infty$.

Consider the orientation equations of $i$-th ODIN:

$$
\left[\begin{array}{c}
\dot{\psi}_{i} \\
\dot{r}_{i}
\end{array}\right]=\left[\begin{array}{cc}
0 & 1 \\
0 & \frac{N_{r}}{I_{z}}
\end{array}\right]\left[\begin{array}{c}
\psi_{i} \\
r_{i}
\end{array}\right]+\left[\begin{array}{c}
0 \\
\frac{1}{I_{z}}
\end{array}\right] \tau_{\psi_{i}} .
$$

The linear state feedback controller for yaw moment is then

$$
\tau_{\psi_{i}}=I_{z}\left(\ddot{\psi}_{i d}-\frac{N_{r}}{I_{z}} \dot{\psi}_{i d}-k_{1}^{\psi_{i}}\left(\psi_{i}-\psi_{i d}\right)-k_{2}^{\psi_{i}}\left(r_{i}-\dot{\psi}_{i d}\right)\right)
$$

where $k_{1}^{\psi_{i}}, k_{2}^{\psi_{i}}>0$ are controller gains. 


\section{Observer design for $\dot{\psi}_{i d}$ and $\ddot{\psi}_{i d}$}

In the equation (50), calculating $\tau_{\psi_{i}}$ requires the values of $\dot{\psi}_{i d}$ and $\ddot{\psi}_{i d}$. But we only have the value of $\psi_{i d}$ according to equation (48). A state observer is designed to estimate $\dot{\psi}_{i d}$ and $\ddot{\psi}_{i d}$ from $\psi_{i d}$.

Define a new state variable $z_{i d}=\dot{r}_{i d}$, then $\dot{z}_{i d}$ is the thirdorder derivative of $\psi_{i d}$ and can be approximated as white Gaussian noise. So we have a system about $\psi_{i d}, \dot{\psi}_{i d}$, and $\ddot{\psi}_{i d}$ as the following:

$$
\left\{\begin{array}{l}
\dot{X}_{\psi_{i}}=A_{\psi} X_{\psi_{i}}+B_{\psi} \omega_{i} \\
\mu_{i}=C_{\psi} X_{\psi_{i}}
\end{array}\right.
$$

where $\omega_{i}$ represents white Gaussian noise ${ }^{1}, \mu_{i}$ denotes the output variable $\psi_{i d}$, and

$$
\begin{aligned}
& X_{\psi_{i}}=\left[\begin{array}{l}
\psi_{i d} \\
r_{i d} \\
z_{i d}
\end{array}\right]=\left[\begin{array}{l}
\psi_{i d} \\
\dot{\psi}_{i d} \\
\ddot{\psi}_{i d}
\end{array}\right] \\
& A_{\psi}=\left[\begin{array}{lll}
0 & 1 & 0 \\
0 & 0 & 1 \\
0 & 0 & 0
\end{array}\right], B_{\psi}=\left[\begin{array}{l}
0 \\
0 \\
1
\end{array}\right], C_{\psi}=\left[\begin{array}{lll}
1 & 0 & 0
\end{array}\right] .
\end{aligned}
$$

Given the value of $\psi_{i d}$, the estimation for $\dot{\psi}_{i d}$ and $\ddot{\psi}_{i d}$ can be obtained by a reduced order Luenberger observer as shown in [18].

\section{SIMULATION RESULTS}

In this section, we carry out simulations to demonstrate the effectiveness of proposed formation controllers. The model parameters of ODIN are taken from [19]: $m=125 \mathrm{~kg}, X_{\dot{u}}=$ $-62.5, Y_{\dot{v}}=-62.5, X_{u}=-100, Y_{v}=-100, N_{r}=-30, I_{z}=$ $8 \mathrm{Nms}^{2}$. We run two simulations.

In the first simulation there are three vehicles which are initialized as follows: $\left(x_{1}, y_{1}\right)=(10 \mathrm{~m}, 8 \mathrm{~m}),\left(x_{2}, y_{2}\right)=$ $(-5 \mathrm{~m}, 10 \mathrm{~m}),\left(x_{3}, y_{3}\right)=(25 \mathrm{~m}, 5 \mathrm{~m}), u_{1}=v_{1}=u_{2}=v_{2}=u_{3}=$ $v_{3}=1 \mathrm{~m} / \mathrm{s}, \quad \psi_{1}=0.1 \mathrm{rad}, \psi_{2}=0.4 \mathrm{rad}, \psi_{3}=0.7 \mathrm{rad}, r_{1}=$ $r_{2}=0.1 \mathrm{rad} / \mathrm{s}$. Suppose the white Gaussian noise in equation (51) is $\mathscr{N}(0,0.1)$. The Jacobi vectors are defined as the equation (20). Let $\rho_{1 d}=(0,10)$ and $\rho_{2 d}=(30,0)$, the desired formation shape is a isosceles triangle. The desired trajectory is a horizontal straight line $y=10$. Simulation results are plotted in Figure 2, Figure 3 and Figure 4. Figure 2 shows the trajectories of the three ODINs. The positions of the three underwater vehicles are marked by ' $D$ ' every 50 seconds. From the Figure 2 we can see that the three ODINs form the triangular formation immediately and keep moving in fixed formation. Figure 3 shows that the surge velocity of the three vehicles converge to $1 \mathrm{~m} / \mathrm{s}$ and the sway velocity converge to 0 . Figure 4 shows how the yaw angles of these vehicles match the desired value and the yaw angle velocities converge to 0 .

In the second simulation, there are six ODINs controlled to simultaneously track the desired trajectory and keep a

\footnotetext{
${ }^{1} \mathrm{~A}$ more rigorous notation can be used to represent the white noise. We adopt the current notation for the sake of simplicity
}

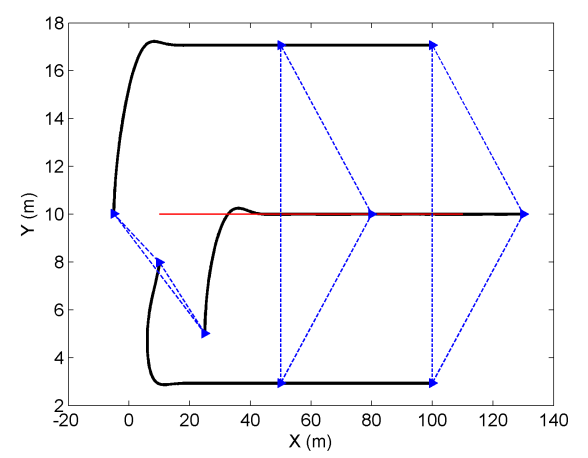

Fig. 2. Three ODINs in a triangular formation moving on a horizontal trajectory.
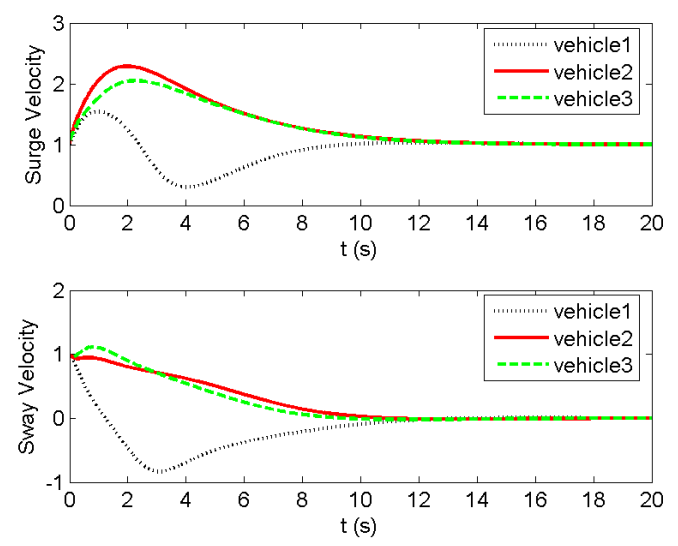

Fig. 3. Surge and sway velocity matching of the ODINs.

polygon formation. The Jacobi vectors are defined by the following equations:

$$
\begin{aligned}
& \rho_{1}=\frac{1}{\sqrt{2}}\left(p_{2}-p_{1}\right), \rho_{2}=\frac{1}{\sqrt{2}}\left(p_{3}-p_{4}\right), \rho_{3}=\frac{1}{\sqrt{2}}\left(p_{5}-p_{6}\right) \\
& \rho_{4}=\frac{1}{2}\left(p_{4}+p_{3}-p_{1}-p_{2}\right) \\
& \rho_{5}=\frac{1}{4}\left(p_{1}+p_{2}+p_{3}+p_{4}-2 p_{5}-2 p_{6}\right) .
\end{aligned}
$$

Let the Jacobi vectors converge to the following desired value:

$$
\begin{aligned}
& \rho_{1} \rightarrow[0,30]^{T}, \rho_{2} \rightarrow[20,20]^{T}, \rho_{3} \rightarrow[-20,20]^{T}, \\
& \rho_{4} \rightarrow[0,0]^{T}, \rho_{5} \rightarrow[0,0]^{T} .
\end{aligned}
$$

It implies that the formation shape is the hexagon plotted in the figure 5.

The desired trajectory of formation center is an sinusoidal line taken as $q_{c d}=[t, 30 * \sin (0.1 t)]^{T}$. Simulation results are plotted in figure 6 . It is seen that all ODINs track their reference trajectories.

\section{CONCLUSIONS AND FEATURE WORKS}

In this paper, we demonstrate a geometric method in formation control of AUVs. Hydrodynamic forces and moments are considered using a 3 DOF horizontal model for 

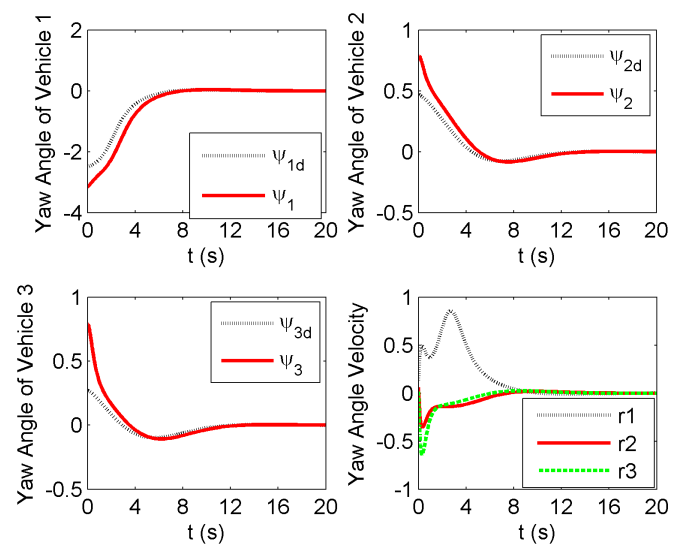

Fig. 4. Yaw angle and velocity matching of the ODINs.

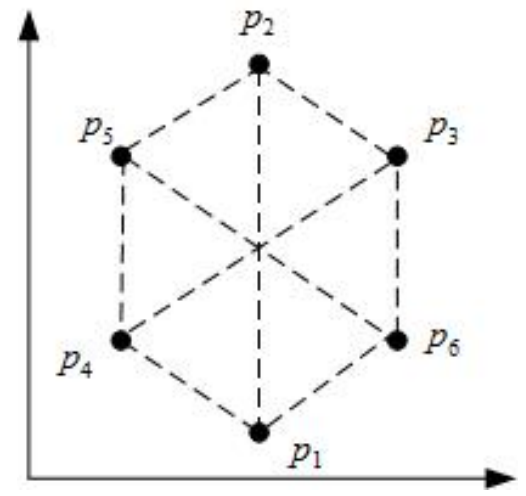

Fig. 5. Formation shape of six ODINs.

each AUV with surge force and yaw moment as control inputs. Jacobi transform is applied to the collected dynamics to reveal the formation shape and the formation center dynamics explicitly. For ODIN vehicle model, the formation shape, formation center and vehicle orientation dynamics are decoupled and linear state feedback controllers are used to stabilize the desired motion. Ongoing work are being developed for the general model of AUVs described by equations(1) and (2).

\section{REFERENCES}

[1] R. Olfati-Saber, J. Fax, and R. M.Murray, "Consensus and cooperation in networked multi-agent systems," Proceedings of the IEEE, vol. 95, pp. 215-233, 2007.

[2] M. Porfiri, D. Roberson, and D. J.Stilwell, "Tracking and formation control of multiple autonomous agents: A two-level consensus approach," Automatica, vol. 43, pp. 1318-1328, 2007.

[3] M. Lewis and K.-H. Tan, "High precision formation control of mobile robots using virtual structures," Autonomous Robots, vol. 4, pp. 387403, 1997.

[4] F. Zhang and N. Leonard, "Generating contour plots using multiple sensor platforms," in Proceedings of IEEE Symposium on Swarm Intelligence, Pasadena,CA, 2005, pp. 309-314.

[5] F. Zhang and N. E. Leonard, "Cooperative control and filtering for cooperative exploration," IEEE Transactions on Automatic Control, vol. Accepted June 2008.

[6] F. Zhang, M. Goldgeier, and P.S.Krishnaprasad, "Control of small formations using shape coordinates," in Proceedings of the 2003 IEEE International Conference on Robotics and Automation, Taipei, Taiwan, 2003, pp. 14-19.

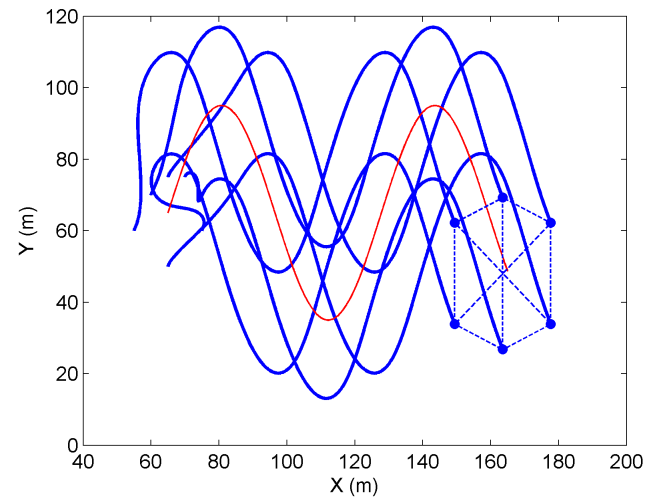

Fig. 6. Six ODINs in a hexagon formation moving on a sinusoidal trajectory.

[7] Q. Jia and G. Li, "Formation control and obstacle avoidance algorithm of multiple autonomous underwater vehicle(AUVs) based on potential function and behavior rules," in Proceedings of IEEE International Conference on Automation and Logistics, Jinan, China, 2007, pp. 569573.

[8] F. Fahimi, "Full formation control for autonomous helicopter groups," Robotica, vol. 26, pp. 143-156, 2008.

[9] S.P.Hou and C.C.Cheah, "PD control scheme for formation control of multiple autonomous underwater vehicles," in IEEE/ASME International Conference on Advanced Intelligent Mechatronics, Singapore, 2009, pp. 356-361.

[10] S.K.Choi, G.Y.Takashige, and J.Yuh, "Experimental study on an underwater robotic vehicle: ODIN," in Proceedings of the 1994 Symposium on Autonomous Underwater Vehicle Technology, Cambridge, Massachusetts, 1994, pp. 79-84.

[11] R. Cui, S. S. Ge, B. V. E. How, and Y. S. Choo, "Leader-follower formation control of underactuated auvs with leader position measurement," in Proceedings of IEEE International Conference on Robotics and Automation, Kobe, Japan, 2009, pp. 979-984.

[12] J. H. Li, "Formation control of multiple underactuated AUVs," in IEEE OCEANS 2007-Europe, Europe, 2007, pp. 1-5.

[13] E. B $\phi$ haug, A. Pavlov, and K. Y. Pettersen, "Cross-track formation control of underactuated autonomous underwater vehicles," Group Coordination and Cooperative Control, vol. 336, pp. 35-54, 2006.

[14] —, "Cross-track formation control of underactuated surface vessels," in Proceedings of the 45th IEEE Conference on Decision and Control, San Diego, CA, 2006, pp. 5955-5961.

[15] D. J. Klein, P. Lee, K. A. Morgansen, and T. Javidi, "Integration of communication and control using discrete time kuramoto models for multivehicle coordination over broadcast networks," in Proceedings of the 46th IEEE Conference on Decision and Control, New Orleans,LA,USA, 2007, pp. 13-19.

[16] D. J. Klein, P. K. Bettale, B. I.Triplett, and K. A. Morgansen, "Autonomous underwater multi-vehicle control with limited communication: Theory and experiment," in Second IFAC Workshop Navigation, Guidance and Control of Underwater Vehicles, Lakeside Hotel, Ireland, 2008.

[17] T. I. Fossen, Gaudiance and Control of Ocean Vehicles, 1st ed. Chichester: John Wiley and Sons, 1994.

[18] P. R. Belanger, Control Engineering: A Mordern Approach. Saunders College Publishing, 1995.

[19] G. Antonelli, Underwater Robots-Motion and Force Control of Vehicle-Manipulator Systems, 2nd ed. Berlin: Springer-Verlag, 2006. 